\title{
Incidence of Cutaneous Melanoma of Eyelid Analysis of the Surveillance, Epidemiology, and End Results Database
}

\author{
Philip Brunetti $^{a}$ Curtis E. Margo ${ }^{a}$ b Dustin D. French ${ }^{c-e}$ \\ a Departments of Ophthalmology, Morsani College of Medicine, University of South Florida, Tampa, FL, USA; \\ ${ }^{b}$ Department of Pathology and Cell Biology, Morsani College of Medicine, University of South Florida, Tampa, FL, \\ USA; ' Department of Ophthalmology, Feinberg School of Medicine, Northwestern University, Chicago, IL, USA; \\ ${ }^{d}$ Center for Health Service and Outcomes Research, Feinberg School of Medicine, Northwestern University, Chicago, \\ IL, USA; ' Veterans Affairs Health Services Research and Development Service, Chicago, IL, USA
}

\section{Keywords}

Eyelid melanoma · Facial melanoma - Melanoma incidence · Ocular oncology $\cdot$ SEER

\begin{abstract}
Purpose: The aim of this work was to report the annual incidence, incidence trend, histological types, and cause-specific survival of cutaneous melanoma of the eyelid from 1975 through to 2017. Methods: Cases were identified in the Surveillance, Epidemiology, and End Results (SEER) database using the ICD-0-3 standard codes for diagnosis and anatomic location. Cutaneous melanomas of the face and scalp/neck were studied as comparison groups. Incidence rates were calculated using the SEER*Stat statistical analysis software with $95 \%$ confidence intervals. Melanoma-specific survival was estimated using the Kaplan-Meier product-limited method. Results: There was an increase in annual incidence of eyelid melanoma over the 43-year study period, ranging from a low of $0.2 \times 10^{6}$ population in 1978 (95\% Cl 0.04-0.6) to a high of $1.0 \times 10^{6}$ population in $2016(95 \% \mathrm{Cl} 2.3-3.5)$. The average annual percent change was 1.2\% (95\% Cl 0.5-1.8). Cause-specific survival of melanoma of the eyelid and facial skin were almost identical (approx. 91.7\%) at 60 months but significantly worse for melanoma of the scalp/neck $(p<$
\end{abstract}

0.05\%). Conclusions: Cutaneous melanoma of the eyelid is uncommon compared to melanoma of facial skin and the scalp/neck. This can be explained in part by the comparatively small surface area at risk. Like melanomas elsewhere, the annual incidence of eyelid melanoma has risen over the last 4 decades, but less than of facial skin and the scalp/neck. Over the span of this study, cause-specific survival from eyelid melanoma was comparable to that of facial skin and better than that of the scalp/neck.

(c) 2020 S. Karger AG, Basel

\section{Introduction}

Most deaths from skin cancers are due to cutaneous melanoma [1]. Depending on the population sample and method of adjustment, the incidence of cutaneous melanoma in North America has increased from 1.8 to $3 \%$ over the last several decades [2-5]. The positive association between increased ultraviolent light exposure, particularly intense intermittent exposure, and risk of cutaneous melanoma is well established. There is relatively limited population-based information on the epidemiology of cutaneous melanoma of the eyelid. Much of the presumed behavior of melanoma at this location is ex-

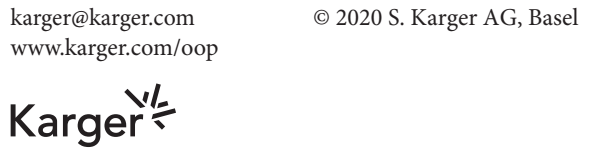


trapolated from experience with cutaneous melanoma in general and from the face in particular [6,7]. We used the Surveillance, Epidemiology, and End Results (SEER) databases to examine the incidence, histological types, and survival outcome of cutaneous melanoma of the eyelid from 1975 through to 2017.

\section{Methods}

Data of patients with cutaneous melanoma of the eyelid were obtained from the case-listing section of the SEER databases of the National Cancer Institute using the SEER 9 registry (1975-2017) and topography code C44.1 for eyelid and the ICD-0-3 Morphology and Behavior codes for melanoma/malignant from $8720 / 3$ through to $8743 / 3$ [8]. Specific morphology codes were employed to identify melanoma subsets: melanoma, nodular (8721); lentigo maligna melanoma $(8741 ; 8742)$; superficial spreading melanoma (8743), and not otherwise specified (8720). Similar codes were used to create 2 comparison groups: melanoma of facial skin (C44.3) and melanoma of scalp and neck (C44.4). Basic demographic information on age, gender, and race were abstracted. Survival analysis included patients with clinical follow-up.

Incidence was age-, race-, and sex-adjusted to the 2000 US standard population. Rates were calculated using SEER*Stat software and reported in cases per million person-years $\left(/ 10^{6}\right)$. Kaplan-Meier survival analyses were performed using melanoma-specific death classification with default SEER*Stat settings for right censoring. For the survival cohort, additional prognostic variables were obtained: Breslow thickness, Clark level of invasion (CS SiteSpecific Factor-1), presence/absence of ulceration, mean mitotic count (CS Site-Specific Factor-7), staging, and site-specific treatment $[9,10]$. SAS ${ }^{\circledR}$ software was used for other statistical analyses. Unless otherwise stated, the $p$ value for tests of statistical inference was $<0.05$. Annual percentage change was calculated using weighted least squares with time as the independent variable. The university IRB exempted this study as the SEER data in the public domain contain no protected personal health information.

\section{Results}

A total of 651 patients with melanoma of eyelid skin were identified in the SEER 9 registry for incidence analysis. The comparison groups included 17,232 patients with melanoma of facial skin and 13,225 patients with melanoma of the scalp/neck. For survival analysis, there were 441 patients with melanoma of eyelid skin, 12,507 with melanoma of facial skin, and 9,769 with melanoma of the scalp/neck.

Patients in the incidence analysis for eyelid melanoma were $98 \%$ white (637) and 344 were men (53\%). In terms of histological types, lentigo maligna melanoma was the most commonly diagnosed $(156,24.24 \%)$, followed by
Table 1. Annual incidence of cutaneous melanoma of the eyelid, face, and scalp/neck, 1975-2017

\begin{tabular}{llcc}
\hline Year & $\begin{array}{l}\text { Eyelid/10 } \\
(95 \% \mathrm{CI})\end{array}$ & $\begin{array}{l}\text { Facial skin } / 10^{6} \\
(95 \% \mathrm{CI})\end{array}$ & $\begin{array}{l}\text { Scalp-neck/106 } \\
(95 \% \mathrm{CI})\end{array}$ \\
\hline 1975 & $0.4(0.1-0.9)$ & $7.7(6.4-9.2)$ & $3.9(3.0-4.9)$ \\
1980 & $0.6(0.2-1.0)$ & $9.6(8.2-11.1)$ & $5.9(4.8-7.1)$ \\
1985 & $0.5(0.3-1.0)$ & $11.8(10.3-13.5)$ & $6.8(5.7-8.0)$ \\
1990 & $0.5(0.2-0.9)$ & $12.0(10.5-13.6)$ & $7.6(6.5-8.9)$ \\
1995 & $0.5(0.3-0.9)$ & $14.6(13.1-16.3)$ & $11.3(10.0-12.8)$ \\
2000 & $0.7(0.4-1.1)$ & $19.0(17.3-20.7)$ & $12.6(11.2-14.0)$ \\
2005 & $0.7(0.4-1.1)$ & $21.6(19.9-23.4)$ & $15.8(14.4-17.4)$ \\
2010 & $0.6(0.5-0.9)$ & $20.3(18.7-22.0)$ & $19.8(18.2-21.5)$ \\
2015 & $0.6(0.4-0.9)$ & $21.5(19.9-23.2)$ & $19.6(18.1-21.2)$ \\
APC, \% & $1.2(0.5-1.8)$ & $2.3(2.1-2.5)$ & $3.9(3.7-4.1)$ \\
\hline
\end{tabular}

Reported at 5-year intervals; a list of the entire 43 years is available from: https://github.com/pbrunetti/eyelid_epidemiology. APC, annual percent change.

superficial spreading melanoma $(142,21.8 \%)$, and nodular melanoma (50,7.7\%). The remaining 303 cases (46.5\%) were either rare subtypes or not otherwise specified.

The incidence of cutaneous eyelid melanoma ranged from a low of $0.2 / 10^{6}$ person-years in 1978 (95\% CI 0.04$\left.0.6 / 10^{6}\right)$ to a high of $1.0 / 10^{6}$ person-years in 2016 (95\% CI $\left.0.7-1.4 / 10^{6}\right)$. Table 1 shows the annual incidence at 5 -year intervals for all racial/origin groups during the 43-year period. There was a small but statistically significant annual upward trend of $1.2 \%$ (95\% CI 0.5-1.8). Larger annual percent changes were found for melanoma of the face $(2.3 \%, 95 \%$ CI $2.1-2.5)$ and scalp/neck $(3.9 \%, 95 \%$ CI 3.7-4.1; Table 1).

Among the 441 patients in the survival cohort, 432 (98\%) were white and 229 (51.9\%) were men. Crude median cause-specific survival at 60 months was $91.7 \%$ (95\% CI 84.4-94.2), compared to $91.7 \%$ (95\% CI 91.2-92.2) for facial skin and $84.3 \%$ (95\% CI 83.5-85.1) for the scalp/ neck (Fig. 1). Breslow thickness, Clark level of invasion, and mitotic counts were available in 197 (44.6\%), 163 (37\%), and $120(27.2 \%)$ patients, respectively. Logistic regression analysis was not performed because multiple critical prognostic variables were missing.

\section{Discussion}

There is limited population-based information on the incidence of melanoma of the eyelid. A study utilizing the Florida Cancer Data System registry in the 1990s revealed 


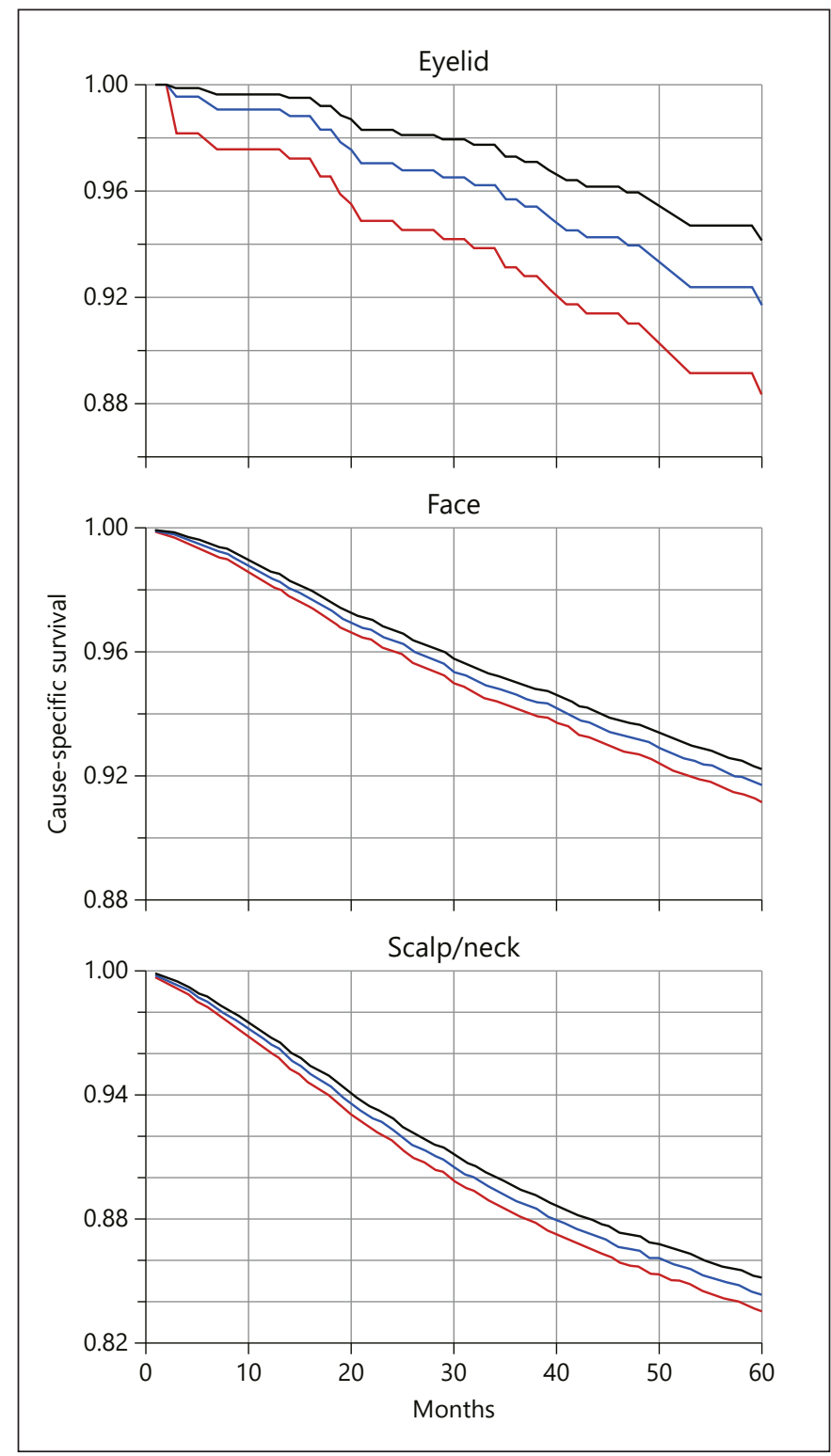

Fig. 1. Kaplan-Meier melanoma-specific survival with 95\% CI for melanoma of eyelid, face, and scalp/neck. Cause-specific survival for melanoma of the face parallels that of the eyelid but has a narrower confidence interval. Survival for eyelid and face was significantly better than for scalp/neck.

an annual incidence of eyelid melanoma of $0.6 / 10^{6}$ personyears among whites over the age of 20 years [11]. The incidence from the Florida Cancer Registry is within the confidence interval obtained in this SEER study during this time period. A population-based study in Sweden from 1976 through to 1994 permitted calculation of a crude incidence rate for eyelid melanoma of $0.4 / 10^{6}$ person-years, also consistent with that observed in this study [12].
Annual incidences in this 43-year survey ranged from a low of $0.2 / 10^{6}$ person-years in 1978 to a high of $1.0 / 10^{6}$ person-years in 2016. It displayed a small overall upward trend. The annual percent change of $1.2 \%$ (95\% CI $0.5-$ 1.8) was less than reported for other skin surfaces (1.8$3 \%$ ) and less than those in the 2 comparison groups (facial melanoma 2.3\%, 95\% CI 2.1-2.5; scalp/neck melanoma $3.9 \%$, 95\% CI 3.7-4.1) [1-6].

We found almost identical cause-specific survival at 5 years for eyelid and facial skin (approx. 91.7\% for both), which was statistically significantly better than survival for melanoma of the scalp/neck $(84.3 \%, p<0.05)$. The better survival of facial melanoma relative to scalp/neck is consistent with what has been described $[13,14]$. To our knowledge, eyelid melanoma-attributed death from a large national sample has not been previously reported. Follow-up was available in 441 patients $(67.7 \%$ of the incidence group). We did not utilize proportional hazard modeling because substantial proportions (often more than half) of the variables associated with outcome were missing.

The low annual incidence of cutaneous melanoma of the eyelid ( $<1$ per million person-years) makes it difficult to accumulate substantial clinical experience with this cancer, even in large referral institutions. By comparison, the overall age-adjusted incidence for cutaneous melanoma in 2011 was 197 cases per million person-years [5]. The low incidence of eyelid melanoma is largely explained by its small surface area, which based on skin surface area calculators ranges from 0.26 to $0.39 \%$ of total skin surface [15]. Other factors such as the use of glasses, sunglasses, eye shields worn in tanning parlors, or the shade cast by brows might also contribute to differences in incidence of melanoma of eyelid compared to other sun-exposed surfaces [6].

The limitations of the SEER registry data have been previously reviewed, the most consistent of which is missing data [16-18]. These limitations are especially problematic in assessing clinical outcome when critical variables associated with tumor-related mortality are absent $[17,18]$. In terms of eyelid melanoma, the success of treatment cannot be meaningfully interpreted without controlling for Breslow thickness (or Clark level), tumor ulceration, and mitotic count. Adding to the problem of missing morphologic variables is incomplete information related to primary treatment, including but not limited to the adequacy of surgical margins and use of adjuvant therapies. 


\section{Conclusions}

The SEER database showed low and slowly increasing annual percentage changes in the incidence of cutaneous melanoma of the eyelid from 1975 through to 2017. Cause-specific survival of melanoma of the eyelid is nearly identical to that of facial melanoma, but better than melanoma of the scalp/neck. Clinical outcome from cancer registries must be interpreted cautiously when substantial proportions of key variables associated with survival cannot be controlled for.

\section{Statement of Ethics}

This study complied with guidelines of human research as it applies to protected health information in medical records. The study was exempted by the Institutional Review Board of the Morsani College of Medicine. Data are available in the public domain and contained no personal medical identifiers.

\section{Conflict of Interest Statement}

The authors have no ethical, proprietary, or commercial conflicts of interest.

\section{Funding Sources}

The study was unfunded.

\section{Author Contributions}

P.B. and C.E.M.: concept/background research. P.B. and D.D.F.: data collection. C.E.M., D.D.F., and P.B.: data analysis. C.E.M.: original manuscript draft. C.E.M., D.D.F., and P.B.: manuscript revisions. C.E.M. and D.D.F.: tables and graphs.

\section{References}

1 Gersehwald JE, Scolyer RA, Hess KR, et al. Melanoma of the skin. In: Amin MD, editor. AJCC Cancer Staging Manual. 8th ed. Chicago; 2017.

2 National Cancer Institute. SEER cancer statistics review, 1975-2013. National Cancer Institute; 2016 . Available from: https://seer.cancer.gov/archive/csr/1975_2013/\#contents.

3 Siegel RL, Miller KD, Jemal A. Cancer statistics. CA Cancer J Clin. 2019;69:7-34.

4 Paulson KG, Gupta D, Kim TS, et al. Age-specific incidence of melanoma in the United States. JAMA Dermatol. 2019;156(1):57-64.

5 Guy GP, Thomas CC, Thompson T, Watson M, Massetti GM, Richardson LC; Centers for Disease Control and Prevention (CDC). Vital signs: melanoma incidence and mortality trends and projection - United States, 1982 - 2030. MMWR Morb Mortal Wkly Rep. 2015 Jun;64(21):591-6.

6 Mancera N, Smalley KS, Margo CE. Melanoma of the eyelid and periocular skin: histopathologic classification and molecular pathology. Surv Ophthalmol. 2019 May-Jun; 64(3):272-88.

7 Margo CE. Pigment lesions of the eyelid. In: Albert DM, Jakobeic FA, editors. Principles and Practice of Ophthalmology. Volume 4. Philadelphia: WB Saunders Company; 2000. pp. 3430-46.
8 National Cancer Institute. Overview of the SEER program. Available from: https://www. seer.cancer.gov/about/overview.html (accessed January, 2018 through July 24, 2020).

9 National Cancer Institute. Skin: Relationship between thickness, depth of invasion and Clark Level. National Cancer Institute SEER; 2020. Available from: https://seer.cancer.gov/ tools/ssm/SSM2018-SKIN.pdf (accessed July 24, 2020).

10 National Cancer Institute. Staging: melanoma of the skin, vulva, penis and scrotum staging. Available from: https://training.seer.cancer. gov/melanoma/abstract-code-stage/staging. html (accessed July 24, 2020).

11 Margo CE, Mulla ZD. Malignant tumors of the eyelid: a population-based study of nonbasal and non-squamous cell malignant neoplasms. Arch Ophthalmol. 1998;116(2):1958.

12 Gillgren P, Mansson-Brahme E, Frisell J, et al. Epidemiologic characteristics of cutaneous malignant melanoma of the head and neck. A population-based study. Acta Oncol. 1999; 38(8):1069-74.
13 Tseng WH, Martinez SR. Tumor location predicts survival in cutaneous head and neck melanoma. J Surg Res. 2011;167(2):192-8.

14 Golger A, Young DS, Ghazarian D, Neligan PC. Epidemiological features and prognostic factors of cutaneous head and neck melanoma: a population-based study. Arch Otolaryngol Head Neck Surg. 2007;133(5):442-7.

15 Prieto MF, Acha B, Gómez-Cía T, et al. A system for $3 \mathrm{D}$ representation of burns and calculation of burnt skin area. Burns. 2011;37(7): 1233-40.

16 Yu JB, Gross CP, Wilson LD, Smith BD. NCI SEER public-use data: applications and limitations in oncology research. Oncology. 2009; 23(3):288-95.

17 Shaikh W, Weinstock MA, Halpern AC, et al. The characterization and potential impact of melanoma cases with unknown thickness in the United States Surveillance, Epidemiology, and End Results Program, 1989-2008. Cancer Epidemiol. 2013;37(1):64-70.

18 Kim HM, Goodman M, Kim BI, et al. Frequency and determinants of missing data in clinical and prognostic variables recently added to SEER. J Registry Manag. 2011;38(3): $120-31$. 